\title{
VALUE FUNCTIONS FOR BOLZA PROBLEMS WITH DISCONTINUOUS LAGRANGIANS AND HAMILTON-JACOBI INEQUALITIES *
}

\author{
Gianni Dal Maso ${ }^{1}$ AND HÉLÈne FrankowskA ${ }^{2}$
}

\begin{abstract}
We investigate the value function of the Bolza problem of the Calculus of Variations

$$
V(t, x)=\inf \left\{\int_{0}^{t} L\left(y(s), y^{\prime}(s)\right) d s+\varphi(y(t)): y \in W^{1,1}\left(0, t ; \mathbb{R}^{n}\right), y(0)=x\right\},
$$

with a lower semicontinuous Lagrangian $L$ and a final $\operatorname{cost} \varphi$, and show that it is locally Lipschitz for $t>0$ whenever $L$ is locally bounded. It also satisfies Hamilton-Jacobi inequalities in a generalized sense. When the Lagrangian is continuous, then the value function is the unique lower semicontinuous solution to the corresponding Hamilton-Jacobi equation, while for discontinuous Lagrangian we characterize the value function by using the so called contingent inequalities.
\end{abstract}

AMS Subject Classification. 49L20, 49L25.

Received July 26, 1999. Revised April 28, 2000.

\section{INTRODUCTION}

Consider the autonomous Bolza minimization problem of the Calculus of Variations

$$
\inf \left\{\int_{0}^{T} L\left(y(s), y^{\prime}(s)\right) d s+\varphi(y(T)) ; y \in W^{1,1}\left(0, T ; \mathbb{R}^{n}\right), y(0)=x\right\}
$$

where $\varphi: \mathbb{R}^{n} \mapsto \mathbb{R}_{+} \cup\{+\infty\}$ and $L: \mathbb{R}^{n} \times \mathbb{R}^{n} \mapsto \mathbb{R}_{+}$are lower semicontinuous and $L(x, \cdot)$ is convex. The classical Lagrange problem (with the fixed final condition $y(T)=x_{T}$ ) may be reduced to the above one by simply setting $\varphi\left(x_{T}\right)=0$ and $\varphi=+\infty$ elsewhere. Lagrange problems were studied in the literature from various points of view. If a Tonelli type coercivity assumption holds true

$$
\exists \Theta: \mathbb{R}^{n} \mapsto \mathbb{R}_{+}, \quad \lim _{|u| \rightarrow \infty} \frac{\Theta(u)}{|u|}=+\infty, \quad \forall(x, u), \quad L(x, u) \geq \Theta(u),
$$

Keywords and phrases: Discontinuous Lagrangians, Hamilton-Jacobi equations, viability theory, viscosity solutions.

* Supported by the Human Capital and Mobility Programme of the European Union, contract number CHRX-CT94-0431.

1 SISSA, via Beirut 2, 34014 Trieste, Italy.

2 CNRS, ERS2064, Centre de Recherche Viabilité, Jeux, Contrôle, Université de Paris-Dauphine, 75775 Paris Cedex 16, France; e-mail: frankows@viab.dauphine.fr 
then absolutely continuous minimizers do exist (see, for instance [13]). Actually the very same condition yields also lower semicontinuity of the value function associated to problem (1.1). It was observed in [2] that, without additional (boundedness) assumptions, minimizers are in general not Lipschitz. This creates a major difficulty in developing the Hamilton-Jacobi theory in a general case. It was also shown in [2] that minimizers are Lipschitz continuous, under the local boundedness condition

$$
\forall R>0, \exists M>0, \exists r>0, \forall(x, u) \in B(0, R) \times B(0, r), \quad L(x, u) \leq M,
$$

where $B(x, r)$ denotes the closed ball with centre $x$ and radius $r$. The above condition may be rewritten in the following equivalent way:

$$
\forall x_{0} \in \mathbb{R}^{n}, \exists M>0, \exists r>0, \forall(x, u) \in B\left(x_{0}, r\right) \times B(0, r), \quad L(x, u) \leq M .
$$

Since any minimizer of problem (1.1) solves also an associated Lagrange problem, the same statement remains valid also for the Bolza problem, whenever the infimum in (1.1) is finite.

Consider the Hamilton-Jacobi equation

$$
\begin{cases}V_{t}+H\left(x,-V_{x}\right)=0 & \text { in } \mathbb{R}_{+}^{\star} \times \mathbb{R}^{n} \\ V(0, \cdot)=\varphi & \text { in } \mathbb{R}^{\mathrm{n}}\end{cases}
$$

where $\mathbb{R}_{+}^{\star}=\mathbb{R}_{+} \backslash\{0\}$ and $H$ is the Hamiltonian associated with $L$, defined by

$$
H(x, p)=\sup _{u \in \mathbb{R}^{n}}(\langle p, u\rangle-L(x, u))
$$

i.e., $H(x, \cdot)$ is the Legendre-Fenchel transform of $L(x, \cdot)$.

It is a well known fact that (1.4) does not have smooth solutions even when $H$ and $\varphi$ are smooth, and one has to use generalized solutions to treat first order equations of the above type. To prove the uniqueness of the solution to (1.4) in the viscosity sense (as done in [15]), the authors assumed, among other hypotheses, the continuity of $H$ and looked for bounded uniformly continuous solutions (in the viscosity sense).

The link between (1.4) and (1.1) is the value function $V: \mathbb{R}_{+} \times \mathbb{R}^{n} \mapsto \mathbb{R}_{+} \cup\{+\infty\}$, defined by

$$
V(t, x)=\inf \left\{\int_{0}^{t} L\left(y(s), y^{\prime}(s)\right) d s+\varphi(y(t)): y \in W^{1,1}\left(0, t ; \mathbb{R}^{n}\right), y(0)=x\right\},
$$

which is, under some regularity assumptions, a viscosity solution to (1.4), i.e., it is a supersolution (resp. subsolution) with derivatives replaced by subdifferentials (resp. superdifferentials). The above definition of value function is somewhat different from the usual one, but, our problem being autonomous, we have found it more convenient for our purposes. The reader accustomed to different definitions can easily make suitable changes to derive similar results for other value functions.

There exists an interplay between subdifferentials of the value function and minimizers. For instance, in Section 3 we show that, if $y$ is a minimizer to the last problem and if the subdifferential of $V$ at $(t, x)$ is nonempty, then the difference quotients $\left\{\frac{y(h)-x}{h}\right\}$ are bounded (Prop. 3.2).

Many results about Bolza problems with smooth data were extended to the case of lower semicontinuous $L$ and $\varphi$. In such case, under Tonelli's assumption (1.2), $H$ is merely upper semicontinuous and, for this reason, it is natural to look for an extension of the viscosity solutions theory to this situation. Also in such general case $V$ is only lower semicontinuous, which creates additional difficulties in formulating maximum principles yielding uniqueness. However we prove that $V$ is locally Lipschitz on $\mathbb{R}_{+}^{\star} \times \mathbb{R}^{n}$, whenever $L$ is locally bounded, even if the data $L$ and $\varphi$ are discontinuous (Cor. 3.4). 
In the case of lower semicontinuous solutions and Hamilton-Jacobi-Bellman equations related to Mayer's problem of optimal control theory, a maximum principle for lower semicontinuous functions was proposed in $[10]$ to deduce uniqueness of solutions to the Hamilton-Jacobi equation (corresponding to Mayer's problem). In $[20,21]$ the same uniqueness result was obtained by exploiting properties of the epigraph of the solutions of the Hamilton-Jacobi equation.

When $L$ is continuous, we prove (Th. 4.1) that $V$ is the unique lower semicontinuous function which satisfies the initial condition $V(0, \cdot)=\varphi$ and solves the Hamilton-Jacobi equation (1.4) in the following sense:

$$
\begin{gathered}
\forall(t, x) \in \operatorname{dom}(V), t>0, \exists u \in \mathbb{R}^{n}, \quad D_{\uparrow} V(t, x)(-1, u) \leq-L(x, u), \\
\forall(t, x) \in \operatorname{dom}(V), \forall u \in \mathbb{R}^{n}, \quad D_{\downarrow} V(t, x)(1,-u) \leq L(x, u)
\end{gathered}
$$

where $D_{\uparrow} V(t, x)$ and $D_{\downarrow} V(t, x)$ are the lower and upper contingent derivatives of $V$ at $(t, x)$, whose definition is recalled in Section 2. We underline that they coincide with Dini's lower and upper derivatives when $V$ is locally Lipschitz.

Contingent inequalities for discontinuous functions were introduced in [3] to study lower semicontinuous Lyapunov functions. They were introduced independently in the context of Lipschitz functions (and Dini's directional derivatives) in [29] to investigate Isaacs' equation of differential games. In [18] contingent inequalities were used to study lower semicontinuous supersolutions of Hamilton-Jacobi-Bellman equation of optimal control.

Under the same assumptions we prove also (Th. 4.5) that the restriction of $V$ to $\mathbb{R}_{+}^{\star} \times \mathbb{R}^{n}$ is the unique locally Lipschitz function which satisfies the initial condition

$$
\liminf _{h \rightarrow 0+, y \rightarrow x} V(h, y)=\varphi(x)
$$

and solves the Hamilton-Jacobi equation (1.4) in the following generalized sense (which is weaker than (1.6) and (1.7)):

$$
\forall(t, x) \in \mathbb{R}_{+}^{\star} \times \mathbb{R}^{n}, \quad \forall\left(p_{t}, p_{x}\right) \in \partial_{-} V(t, x), p_{t}+H\left(x,-p_{x}\right)=0,
$$

where $\partial_{-} V(t, x)$ denotes the subdifferential of $V$ at $(t, x)$.

Moreover we prove that the restriction of $V$ to $\mathbb{R}_{+}^{\star} \times \mathbb{R}^{n}$ is the unique locally Lipschitz function which satisfies the initial condition (1.8) and solves the Hamilton-Jacobi equation (1.4) in the viscosity sense:

$$
\begin{aligned}
& \forall(t, x) \in \mathbb{R}_{+}^{\star} \times \mathbb{R}^{n}, \quad \forall\left(p_{t}, p_{x}\right) \in \partial_{-} V(t, x), \quad p_{t}+H\left(x,-p_{x}\right) \geq 0, \\
& \forall(t, x) \in \mathbb{R}_{+}^{\star} \times \mathbb{R}^{n}, \quad \forall\left(p_{t}, p_{x}\right) \in \partial_{+} V(t, x), \quad p_{t}+H\left(x,-p_{x}\right) \leq 0,
\end{aligned}
$$

where $\partial_{+} V(t, x)$ denotes the superdifferential of $V$ at $(t, x)$. We have been not able to prove that there is only one lower semicontinuous viscosity solution. For this reason we have to adopt a rather unusual notion of solution in our uniqueness result for lower semicontinuous solutions.

When $L$ is discontinuous and locally bounded, we prove (Th. 5.1) that $V$ is the unique lower semicontinuous function which satisfies the initial condition $V(0, \cdot)=\varphi$ and solves the Hamilton-Jacobi equation (1.4) in the following sense:

$$
\begin{gathered}
\forall(t, x) \in \operatorname{dom}(V), t>0, \exists u \in \mathbb{R}^{n}, \quad D_{\uparrow} V(t, x)(-1, u) \leq-L(x, u), \\
\forall(t, x) \in \operatorname{dom}(V), \forall u \in \mathbb{R}^{n}, \quad D_{\downarrow} V(t, x)(1,-u) \leq L^{+}(x, u),
\end{gathered}
$$


where $L^{+}$is defined by

$$
L^{+}(x, u)=\limsup _{h \rightarrow 0+} \frac{1}{h} \inf \left\{\int_{-h}^{0} L\left(y(s), y^{\prime}(s)\right) d s: y(-h)=x-h u, y(0)=x\right\}
$$

(see [16] and [1]).

Under the same hypotheses we prove also (Th. 5.5) that the restriction of $V$ to $\mathbb{R}_{+}^{\star} \times \mathbb{R}^{n}$ is the unique locally Lipschitz function which satisfies the initial condition (1.8) together with the additional condition

$$
\forall x \in \mathbb{R}^{n}, \forall \lambda>0, \lim _{\substack{h \rightarrow 0+, y \rightarrow x \\|y-x| \leq \lambda h}} V(h, y)=\varphi(x),
$$

and solves the Hamilton-Jacobi equation (1.4) in the following sense:

$$
\begin{gathered}
\forall(t, x) \in \mathbb{R}_{+}^{\star} \times \mathbb{R}^{n}, \quad \forall\left(p_{t}, p_{x}\right) \in \partial_{-} V(t, x), p_{t}+H\left(x,-p_{x}\right) \geq 0, \\
\forall(t, x) \in \mathbb{R}_{+}^{\star} \times \mathbb{R}^{n}, \quad \forall u \in \mathbb{R}^{n}, \quad D_{\downarrow} V(t, x)(1,-u) \leq L^{+}(x, u) .
\end{gathered}
$$

In all these theorems, the uniqueness is obtained by proving suitable comparison results for the corresponding notions of sub- and supersolution. In particular, we extend here a result from [19] proved in the context of Mayer's problem with bounded dynamics, and show (Cor. 5.3) that, if (1.3) holds true, then the value function is the smallest lower semicontinuous function satisfying the initial condition $V(0, \cdot)=\varphi$ and the contingent inequality (1.6).

Recently solutions to the Hamilton-Jacobi equation of a nonautonomous Bolza problem were investigated in [23]. However the results of [23] do not overlap with ours, since the assumptions of that article imply that the Hamiltonian is locally Lipschitz.

\section{Preliminaries}

Let $K \subset \mathbb{R}^{n}$ be a nonempty subset and $x \in K$. The contingent cone $T_{K}(x)$ to $K$ at $x$ is defined by

$$
v \in T_{K}(x) \Longleftrightarrow \liminf _{h \rightarrow 0+} \frac{\operatorname{dist}(x+h v, K)}{h}=0 .
$$

The negative polar cone $T^{-}$to a subset $T \subset \mathbb{R}^{n}$ is given by

$$
T^{-}=\left\{v \in \mathbb{R}^{n}: \forall w \in T,\langle v, w\rangle \leq 0\right\} .
$$

We recall the following result due to Cornet [14] (see also [9]).

Theorem 2.1. Let $K \subset \mathbb{R}^{n}$ be a closed subset and $x \in K$. Then

$$
\operatorname{Liminf}_{\substack{y \rightarrow x \\ y \in K}} \overline{c o} T_{K}(y)=C_{K}(x) \subset T_{K}(x),
$$

where Liminf denotes the topological lower limit (in the Painlevé-Kuratowski sense) and $C_{K}(x)$ denotes Clarke's tangent cone to $K$ at $x$.

Let $W: \mathbb{R}^{n} \rightarrow \mathbb{R} \cup\{+\infty\}$ be a lower semicontinuous function. The subdifferential of $W$ at $x \in \operatorname{dom}(W)$ is defined by

$$
\partial_{-} W(x)=\left\{p \in \mathbb{R}^{n}: \liminf _{y \rightarrow x} \frac{W(y)-W(x)-\langle p, y-x\rangle}{|y-x|} \geq 0\right\}
$$


By [19] (or [9])

$$
p \in \partial_{-} W(x) \Longleftrightarrow(p,-1) \in\left[T_{\mathcal{E} p i(W)}(x, W(x))\right]^{-},
$$

where $\mathcal{E} p i(W)$ denotes the epigraph of $W$ defined by

$$
\mathcal{E} p i(W):=\left\{(x, r) \in \mathbb{R}^{n} \times \mathbb{R}: r \geq W(x)\right\} .
$$

An equivalent definition of subdifferential uses directional derivatives of $W$ defined by

$$
\forall u \in \mathbb{R}^{n}, \quad D_{\uparrow} W(x)(u)=\liminf _{\substack{h \rightarrow 0+\\ v \rightarrow u}} \frac{W(x+h v)-W(x)}{h} .
$$

Clearly for every $x \in \operatorname{dom}(W)$

$$
\mathcal{E} p i\left(D_{\uparrow} W(x)(\cdot)\right)=T_{\mathcal{E} p i(W)}(x, W(x))
$$

and therefore

$$
p \in \partial_{-} W(x) \Longleftrightarrow \forall v \in \mathbb{R}^{n},\langle p, v\rangle \leq D_{\uparrow} W(x)(v)
$$

The upper directional derivative of $W$ at $x$ in the direction $u$ is defined by

$$
\forall u \in \mathbb{R}^{n}, \quad D_{\downarrow} W(x)(u)=\limsup _{\substack{h \rightarrow 0+\\ v \rightarrow u}} \frac{W(x+h v)-W(x)}{h} .
$$

The superdifferential $\partial_{+} W(x)$ of $W$ at $x$ is defined by $\partial_{+} W(x)=-\partial_{-}(-W)(x)$ or, equivalently, by

$$
p \in \partial_{+} W(x) \Longleftrightarrow \forall v \in \mathbb{R}^{n},\langle p, v\rangle \geq D_{\downarrow} W(x)(v) .
$$

We shall need the following version of Rockafellar's result (see [27]).

Lemma 2.2. Let $x \in \operatorname{dom}(W)$ and let $(p, 0) \in\left[T_{\mathcal{E}_{p i(W)}}(x, W(x))\right]^{-}$be such that $p \neq 0$. Then there exist $x_{\varepsilon}$ converging to $x$ (as $\varepsilon \rightarrow 0+$ ) and

$$
\left(p_{\varepsilon}, q_{\varepsilon}\right) \in\left[T_{\mathcal{E}_{p i(W)}}\left(x_{\varepsilon}, W\left(x_{\varepsilon}\right)\right)\right]^{-}
$$

converging to $(p, 0)$ as $\varepsilon \rightarrow 0+$ such that $q_{\varepsilon}<0$.

A closed subset $K$ of $\mathbb{R}^{n}$ is called a viability domain of a set-valued map $G: \mathbb{R}^{n} \rightsquigarrow \mathbb{R}^{n}$ if for every $x \in K$

$$
G(x) \cap T_{K}(x) \neq \emptyset .
$$

The following formulation summarizes several versions of the viability theorem (see [4] and [5]).

Theorem 2.3 (Viability). Suppose that $G: \mathbb{R}^{n} \rightsquigarrow \mathbb{R}^{n}$ is an upper semicontinuous set-valued map with compact convex values. For a closed subset $K \subset \mathbb{R}^{n}$ the following conditions are equivalent:

(a) $K$ is a viability domain of $G$;

(b) $G(x) \cap \overline{c o} T_{K}(x) \neq \emptyset$ for every $x \in K$;

(c) for every $x_{0} \in K$ there exist $\varepsilon>0$ and a solution $x:[0, \varepsilon[\mapsto K$ to the Cauchy problem 


$$
\left\{\begin{array}{l}
x^{\prime}(t) \in G(x(t)) \\
x(0)=x_{0}
\end{array}\right.
$$

The equivalence $(\mathrm{a}) \Longleftrightarrow(\mathrm{b})$ was proved in [24]. This proof was simplified in [5] (p. 85). The fact that (a) $\Longleftrightarrow$ (c) was first proved by Bebernes and Schuur in [11]. A proof can be found in [7] or [4,5].

\section{The value function of the Bolza problem}

Consider a lower semicontinuous function $L: \mathbb{R}^{n} \times \mathbb{R}^{n} \mapsto \mathbb{R}_{+}$and an extended lower semicontinuous function $\varphi: \mathbb{R}^{n} \mapsto \mathbb{R}_{+} \cup\{+\infty\}$, not identically equal to $+\infty$. Throughout the whole paper we suppose that $L$ is convex in the second variable and that the coercivity assumption (1.2) holds true. Without any loss of the generality we also assume that $\Theta$ is convex.

Given $t_{0}>0$ and $x_{0} \in \mathbb{R}^{n}$, let us consider the Bolza problem:

$$
\operatorname{minimize} \int_{0}^{t_{0}} L\left(y(s), y^{\prime}(s)\right) d s+\varphi\left(y\left(t_{0}\right)\right)
$$

over all absolutely continuous functions $y \in W^{1,1}\left(0, t_{0} ; \mathbb{R}^{n}\right)$ satisfying the initial condition $y(0)=x_{0}$. The dynamic programming approach associates with this problem the family of problems $\left(t \geq 0, x \in \mathbb{R}^{n}\right)$ :

$$
\operatorname{minimize} \int_{0}^{t} L\left(y(s), y^{\prime}(s)\right) d s+\varphi(y(t))
$$

over all absolutely continuous functions $y \in W^{1,1}\left(0, t ; \mathbb{R}^{n}\right)$ satisfying $y(0)=x$. The value function $V: \mathbb{R}_{+} \times \mathbb{R}^{n} \mapsto$ $\mathbb{R}_{+} \cup\{+\infty\}$ is defined by

$$
V(t, x)=\inf \left\{\int_{0}^{t} L\left(y(s), y^{\prime}(s)\right) d s+\varphi(y(t)): y(0)=x\right\}
$$

Proposition 3.1. Under the above assumptions for all $(t, x) \in \mathbb{R}_{+} \times \mathbb{R}^{n}$ the infimum in (3.1) is attained (it may be infinite) and $V$ is lower semicontinuous on $\mathbb{R}_{+} \times \mathbb{R}^{n}$. Furthermore, if $L$ is locally bounded, then $V$ has finite values on $\mathbb{R}_{+}^{\star} \times \mathbb{R}^{n}$ and satisfies (1.8) and (1.9).

Proof. The existence of a minimizer is a well known result. For the reader's convenience, we sketch the proof of the lower semicontinuity of $V$. Consider a sequence $\left(t_{i}, x_{i}\right) \in \mathbb{R}_{+} \times \mathbb{R}^{n}$ converging to $(t, x)$ such that $V\left(t_{i}, x_{i}\right)$ converge to $\liminf (s, y) \rightarrow(t, x) V(s, y)$. Let $y_{i}$ be the corresponding minimizers of (3.1) with $(t, x)$ replaced by $\left(t_{i}, x_{i}\right)$. If $\lim _{i \rightarrow+\infty} V\left(t_{i}, x_{i}\right)=+\infty$, then

$$
V(t, x) \leq \lim _{i \rightarrow+\infty} V\left(t_{i}, x_{i}\right) .
$$

Assume next that the above limit is finite. Hence for some $M$ for all $i$,

$$
\int_{0}^{t_{i}} L\left(y_{i}(s), y_{i}^{\prime}(s)\right) d s \leq M
$$

Set $y_{i}(s)=y_{i}\left(t_{i}\right)$ for $s \geq t_{i}$. By the coercivity assumption (1.2), the sequence $y_{i}^{\prime}$ is equiintegrable on [0,t +1$]$. This, the Dunford-Pettis criterion (see, e.g. [17], Th. II.25), and the Ascoli-Arzelà theorem yield the existence 
of a subsequence $y_{i_{k}}$ converging uniformly to some $y$ on $[0, t+1]$ such that $y_{i_{k}}^{\prime}$ converge to $y^{\prime}$ weakly in $L^{1}$. We denote this subsequence again by $y_{i}$. Then $y(0)=x$ and $y_{i}\left(t_{i}\right) \rightarrow y(t)$. Fix $0<\varepsilon<t$. Then for all large $i$,

$$
\left.\left.\int_{0}^{t_{i}} L\left(y_{i}(s), y_{i}^{\prime}(s)\right)\right) d s \geq \int_{0}^{t-\varepsilon} L\left(y_{i}(s), y_{i}^{\prime}(s)\right)\right) d s .
$$

Since $L$ is lower semicontinuous and convex in the second variable, from the lower semicontinuity theorems by Olech [26] and Ioffe [25] (see also [12], Th. 2.3.3) it follows that

$$
\left.\liminf _{i \rightarrow \infty} \int_{0}^{t-\varepsilon} L\left(y_{i}(s), y_{i}^{\prime}(s)\right)\right) d s \geq \int_{0}^{t-\varepsilon} L\left(y(s), y^{\prime}(s)\right) d s .
$$

Thus

$$
\left.\liminf _{i \rightarrow \infty} \int_{0}^{t_{i}} L\left(y_{i}(s), y_{i}^{\prime}(s)\right)\right) d s \geq \int_{0}^{t-\varepsilon} L\left(y(s), y^{\prime}(s)\right) d s
$$

Since $\int_{0}^{t-\varepsilon} L\left(y(s), y^{\prime}(s)\right) d s$ converges to $\int_{0}^{t} L\left(y(s), y^{\prime}(s)\right) d s$ when $\varepsilon \rightarrow 0+$, the lower semicontinuity of $\varphi$ yields lower semicontinuity of $V$.

Since $\varphi$ is not identically $+\infty$, it is clear that $V$ is finite on $\mathbb{R}_{+}^{\star} \times \mathbb{R}^{n}$ whenever $L$ is locally bounded.

To prove $(1.9)$ consider a sequence $\left(h_{i}, x_{i}\right) \rightarrow(0+, x)$ such that $\left(x_{i}-x\right) / h_{i}$ is bounded. Then, by lower semicontinuity of $V$,

$$
\begin{gathered}
\liminf _{i \rightarrow \infty} V\left(h_{i}, x_{i}\right) \geq V(0, x)=\varphi(x), \\
V\left(h_{i}, x_{i}\right) \leq \varphi(x)+\int_{0}^{h_{i}} L\left(x_{i}+s\left(x-x_{i}\right) / h_{i},\left(x-x_{i}\right) / h_{i}\right) d s .
\end{gathered}
$$

Passing to the upper limit when $i \rightarrow \infty$, and using the fact that $L$ is locally bounded, we obtain (1.9). Condition (1.8) follows now from (1.9) and from the lower semicontinuity of $V$.

Proposition 3.2. Let $(t, x) \in \operatorname{dom}(V)$, with $t>0$, and let $y$ be a minimizer of (3.1). Assume either that $\partial_{-} V(t, x)$ is nonempty or that (1.3) holds. Then the set

$$
\left\{\frac{y(h)-x}{h}\right\}_{h>0}
$$

is bounded in $\mathbb{R}^{n}$. Furthermore, if

$$
\frac{y\left(h_{i}\right)-x}{h_{i}} \rightarrow u
$$

for some $h_{i} \rightarrow 0+$, then

$$
D_{\uparrow} V(t, x)(-1, u) \leq-L(x, u) .
$$

Consequently, $V$ is a viscosity supersolution to (1.4), i.e.,

$$
\forall(t, x) \in \mathbb{R}_{+}^{\star} \times \mathbb{R}^{n}, \quad \forall\left(p_{t}, p_{x}\right) \in \partial_{-} V(t, x), p_{t}+H\left(x,-p_{x}\right) \geq 0 .
$$


Proof. First we observe that for all $0 \leq h \leq t$

$$
V(t, x)=V(t-h, y(h))+\int_{0}^{h} L\left(y(s), y^{\prime}(s)\right) d s .
$$

Step 1. Let us prove that (3.2) is bounded. If (1.3) holds, then (3.2) follows from [2]. Suppose now that $\partial_{-} V(t, x)$ is nonempty. If $(3.2)$ does not hold, then there exists a sequence $h_{i} \rightarrow 0+$ such that

$$
\frac{\left|y\left(h_{i}\right)-x\right|}{h_{i}} \rightarrow+\infty
$$

Taking a subsequence, still denoted by $h_{i}$, we may assume that for some $v \in \mathbb{R}^{n}$

$$
\frac{y\left(h_{i}\right)-x}{\left|y\left(h_{i}\right)-x\right|} \rightarrow v
$$

Fix $\left(p_{t}, p_{x}\right) \in \partial_{-} V(t, x)$. By the definition of subdifferential

$$
\liminf _{h \rightarrow 0+} \frac{V(t-h, y(h))-V(t, x)-\left\langle\left(p_{t}, p_{x}\right),(-h, y(h)-x)\right\rangle}{h+|y(h)-x|} \geq 0,
$$

which yields, by (3.6),

$$
\limsup _{i \rightarrow \infty} \frac{1}{h_{i}+\left|y\left(h_{i}\right)-x\right|} \int_{0}^{h_{i}} L\left(y(s), y^{\prime}(s)\right) d s \leq-\left\langle p_{x}, v\right\rangle .
$$

By (1.2) this inequality implies that there exists a constant $c>0$ such that

$$
\forall i \in \mathbb{N}, \frac{1}{h_{i}} \int_{0}^{h_{i}} \Theta\left(y^{\prime}(s)\right) d s \leq c\left(1+\frac{\left|y\left(h_{i}\right)-x\right|}{h_{i}}\right) .
$$

By Jensen's inequality we obtain

$$
\forall i \in \mathbb{N}, \quad \Theta\left(\frac{y\left(h_{i}\right)-x}{h_{i}}\right) \leq c\left(1+\frac{\left|y\left(h_{i}\right)-x\right|}{h_{i}}\right) .
$$

By (3.7) this contradicts the coercivity assumption (1.2) and ends the proof of our claim.

Step 2. Let us fix $R>0$ and $\varepsilon>0$. We want to prove that there exists $\delta>0$ such that

$$
\forall \xi \in B(x, \delta), \quad \forall u \in B(0, R), \quad L(\xi, u) \geq L(x, u)-\varepsilon
$$

We start by observing that the function $L(x, \cdot)$ is continuous, since it is convex and finite valued. Thus for every $v \in B(0, R)$ there exists $\rho_{1}=\rho_{1}(v, \varepsilon)>0$ such that

$$
\forall u \in B\left(v, \rho_{1}\right), \quad L(x, v) \geq L(x, u)-\frac{\varepsilon}{2} .
$$

As $L$ is lower semicontinuous, for every $v \in B(0, R)$ there exists $\rho_{2}=\rho_{2}(v, \varepsilon)>0$ such that

$$
\forall \xi \in B\left(x, \rho_{2}\right), \quad \forall u \in B\left(v, \rho_{2}\right), \quad L(\xi, u) \geq L(x, v)-\frac{\varepsilon}{2} .
$$


Putting $r=r(v, \varepsilon)=\min \left\{\rho_{1}(v, \varepsilon), \rho_{2}(v, \varepsilon)\right\}$, it follows that

$$
\forall \xi \in B(x, r), \quad \forall u \in B(v, r), \quad L(\xi, u) \geq L(x, u)-\varepsilon .
$$

By compactness there exists a finite set $\left\{v_{1}, \ldots, v_{k}\right\}$ contained in $B(0, R)$ such that $B(0, R) \subset B\left(v_{1}, r_{1}\right) \cup \cdots \cup$ $B\left(v_{k}, r_{k}\right)$, where $r_{i}=r\left(v_{i}, \varepsilon\right)$. It is then clear that $(3.9)$ is satisfied with $\delta=\min \left\{r_{1}, \ldots, r_{k}\right\}$.

Step 3. Consider now a sequence $h_{i} \rightarrow 0+$ and $u \in \mathbb{R}^{n}$ such that (3.3) holds. By (3.6) we have

$$
D_{\uparrow} V(t, x)(-1, u) \leq-\limsup _{i \rightarrow \infty} \frac{1}{h_{i}} \int_{0}^{h_{i}} L\left(y(s), y^{\prime}(s)\right) d s .
$$

From (3.6) and (3.8) we obtain

$$
\limsup _{i \rightarrow \infty} \frac{1}{h_{i}} \int_{0}^{h_{i}} L\left(y(s), y^{\prime}(s)\right) d s \leq p_{t}-\left\langle p_{x}, u\right\rangle .
$$

By (1.2) this implies that there exists a constant $C>0$ such that

$$
\forall i \in \mathbb{N}, \quad \frac{1}{h_{i}} \int_{0}^{h_{i}} \Theta\left(y^{\prime}(s)\right) d s \leq C .
$$

Let us fix $R>0$ and $\varepsilon>0$. By (3.9) for $i$ large enough we have

$$
L\left(y(s), y^{\prime}(s)\right) \geq L\left(x, y^{\prime}(s)\right)-\varepsilon
$$

for every $s \in\left[0, h_{i}\right]$ such that $\left|y^{\prime}(s)\right|<R$. For every $i$ let

$$
A_{i}=\left\{s \in\left[0, h_{i}\right]:\left|y^{\prime}(s)\right|<R\right\}, \quad B_{i}=\left\{s \in\left[0, h_{i}\right]:\left|y^{\prime}(s)\right| \geq R\right\},
$$

and let $\theta(R)=\min \{\Theta(v):|v| \geq R\}$. Then

$$
\left|B_{i}\right| \leq \frac{1}{\theta(R)} \int_{0}^{h_{i}} \Theta\left(y^{\prime}(s)\right) d s \leq \frac{C}{\theta(R)} h_{i},
$$

where $|\cdot|$ denotes the Lebesgue measure. Consequently $\left|A_{i}\right| \geq \tau(R) h_{i}$, where

$$
\tau(R)=\left(1-\frac{C}{\theta(R)}\right) \rightarrow 1 \text { as } R \rightarrow+\infty
$$

By (3.12) we have

$$
\frac{1}{h_{i}} \int_{0}^{h_{i}} L\left(y(s), y^{\prime}(s)\right) d s \geq \frac{\tau(R)}{\left|A_{i}\right|} \int_{A_{i}} L\left(x, y^{\prime}(s)\right) d s-\varepsilon
$$

and by Jensen's inequality we obtain

$$
\frac{1}{h_{i}} \int_{0}^{h_{i}} L\left(y(s), y^{\prime}(s)\right) d s \geq \tau(R) L\left(x, u_{i}\right)-\varepsilon
$$


where $u_{i}=\left|A_{i}\right|^{-1} \int_{A_{i}} y^{\prime}(s) d s$. For every $v \in \mathbb{R}^{n}$ let $\omega(v)=|L(x, u+v)-L(x, u)|$. Since $L(x, \cdot)$ is continuous at $u$, the function $\omega$ is continuous at 0 and $\omega(0)=0$. From (3.13) we have

$$
\frac{1}{h_{i}} \int_{0}^{h_{i}} L\left(y(s), y^{\prime}(s)\right) d s \geq \tau(R) L(x, u)-\omega\left(u_{i}-u\right)-\varepsilon
$$

In order to estimate $\left|u_{i}-u\right|$, we notice that

$$
\left|u_{i}-u\right| \leq\left|\frac{1}{\left|A_{i}\right|} \int_{A_{i}} y^{\prime}(s) d s-\frac{1}{h_{i}} \int_{0}^{h_{i}} y^{\prime}(s) d s\right|+\varepsilon_{i},
$$

where

$$
\varepsilon_{i}=\left|\frac{y\left(h_{i}\right)-x}{h_{i}}-u\right| \rightarrow 0 \text { as } i \rightarrow \infty
$$

Therefore

$$
\left|u_{i}-u\right| \leq \frac{1}{\left|A_{i}\right|} \int_{B_{i}}\left|y^{\prime}(s)\right| d s+\left(\frac{1}{\left|A_{i}\right|}-\frac{1}{h_{i}}\right) \int_{0}^{h_{i}}\left|y^{\prime}(s)\right| d s+\varepsilon_{i} .
$$

Let us define

$$
\zeta(R)=\min _{|v| \geq R} \frac{\Theta(v)}{|v|}
$$

By $(1.2) \zeta(R) \rightarrow+\infty$ as $R \rightarrow+\infty$, and there exists a constant $\alpha \geq 0$ such that $\Theta(v) \geq|v|-\alpha$ for every $v \in \mathbb{R}^{n}$. From (3.15) we obtain

$$
\begin{aligned}
\left|u_{i}-u\right| & \leq\left(\frac{1}{\tau(R) \zeta(R)}+\frac{1}{\tau(R)}-1\right) \frac{1}{h_{i}} \int_{0}^{h_{i}} \Theta\left(y^{\prime}(s)\right) d s+\alpha\left(\frac{1}{\tau(R)}-1\right)+\varepsilon_{i} \\
& \leq \frac{C}{\tau(R) \zeta(R)}+(C+\alpha)\left(\frac{1}{\tau(R)}-1\right)+\varepsilon_{i}
\end{aligned}
$$

Since $\tau(R) \rightarrow 1$ and $\zeta(R) \rightarrow+\infty$ as $R \rightarrow+\infty$, we have

$$
\left|u_{i}-u\right| \leq \sigma(R)+\varepsilon_{i},
$$

where $\sigma(R) \rightarrow 0$ as $R \rightarrow+\infty$. Therefore we deduce from (3.10) and (3.14) that

$$
D_{\uparrow} V(t, x)(-1, u) \leq-\tau(R) L(x, u)+\omega+\varepsilon,
$$

for every $R>0$ and $\varepsilon>0$. Taking the limit as $R \rightarrow \infty$ and $\varepsilon \rightarrow 0+$ we obtain (3.4).

Step 4. Let us prove (3.5). Pick $\left(p_{t}, p_{x}\right) \in \partial_{-} V(t, x)$ and $u$ satisfying (3.3). From (2.4) and (3.4) we get $p_{t}+\left\langle-p_{x}, u\right\rangle-L(x, u) \geq 0$. The conclusion follows from the definition (1.5) of $H$.

Proposition 3.3. Assume that $L$ is locally bounded and let $\left(t_{0}, x_{0}\right) \in \mathbb{R}_{+}^{\star} \times \mathbb{R}^{n}$. Then there exist $r>0$ and $\delta>0$ such that for all $(t, x) \in B\left(\left(t_{0}, x_{0}\right), \delta\right)$ every minimizer $y(\cdot ; t, x)$ of (3.1) is $r$-Lipschitz. 
Proof. According to [2] (Th. 4.1) for every minimizer $y(\cdot):=y(\cdot ; t, x)$ of $(3.1)$ there exists $k(t, x)$ such that for some $p(s) \in \partial_{u} L\left(y(s), y^{\prime}(s)\right)$

$$
\left\langle p(s), y^{\prime}(s)\right\rangle-L\left(y(s), y^{\prime}(s)\right)=k(t, x) \text { for a.e. } s \in[0, t]
$$

On the other hand, since $L$ is locally bounded, for some $M_{1}>0$ and for all $(t, x)$ sufficiently close to $\left(t_{0}, x_{0}\right)$

$$
\int_{0}^{t} L\left(y(s ; t, x), y^{\prime}(s ; t, x)\right) d s \leq M_{1}
$$

This and coercivity assumption $(1.2)$ imply that $y^{\prime}(\cdot ; t, x)$ are equiintegrable and therefore $y(\cdot ; t, x)$ is uniformly bounded in $L^{\infty}$ for $(t, x)$ near $\left(t_{0}, x_{0}\right)$. Furthermore, there exists $R>0$ such that for all $(t, x)$ sufficiently close to $\left(t_{0}, x_{0}\right)$ the sets

$$
A(t, x):=\left\{s \in[0, t]:\left|y^{\prime}(s ; t, x)\right| \leq R\right\}
$$

have positive measure. Since $L$ is locally bounded,

$$
\sup _{s \in[0, t]} \sup _{u \in B(0,2 R)} L(y(s ; t, x), u) \leq M_{2}<\infty
$$

for all $(t, x)$ sufficiently close to $\left(t_{0}, x_{0}\right)$. This implies that for some $M_{3}<+\infty L(y(s ; t, x), \cdot)$ is $M_{3}-\operatorname{Lipschitz}$ on $B(0, R)$ for $(t, x)$ near $\left(t_{0}, x_{0}\right)$ (see, e.g. [6], proof of Th. 2.1). Hence for almost every $s \in A(t, x)$ we have $\left|\left\langle p(s), y^{\prime}(s)\right\rangle\right| \leq M_{3} R$, which implies

$$
|k(t, x)| \leq M_{2}+M_{3} R
$$

Consequently, $k(t, x)$ is bounded in a neighborhood of $\left(t_{0}, x_{0}\right)$. By [2] (proof of Th. 4.2), $y(\cdot ; t, x)$ are Lipschitz with the same Lipschitz constant whenever $(t, x)$ is sufficiently close to $\left(t_{0}, x_{0}\right)$.

Corollary 3.4. If $L$ is locally bounded, then $V$ is locally Lipschitz on $\mathbb{R}_{+}^{\star} \times \mathbb{R}^{n}$.

Proof. Fix $\left(t_{0}, x_{0}\right) \in \mathbb{R}_{+}^{\star} \times \mathbb{R}^{n} \subset \operatorname{dom}(V)$. By Proposition 3.3, there exist $r>0$ and $\delta>0$ such that for all $(t, x) \in B\left(\left(t_{0}, x_{0}\right), \delta\right)$ every minimizer $y(\cdot ; t, x)$ of $(3.1)$ is $r$-Lipschitz. We may assume that $5 \delta<t_{0}$. Let $\left(t_{1}, x_{1}\right)$ and $\left(t_{2}, x_{2}\right)$ be two distinct points of $B\left(\left(t_{0}, x_{0}\right), \delta\right)$, let $h_{1}=\left|t_{1}-t_{2}\right|+\left|x_{1}-x_{2}\right|$, and $s_{1}=h_{1}-t_{1}+t_{2}$. Let $u_{1} \in \mathbb{R}^{n}$ be such that $y\left(s_{1} ; t_{2}, x_{2}\right)=x_{1}+h_{1} u_{1}$. Then $0<h_{1}<t_{1}, 0 \leq s_{1} \leq 2 h_{1}$, and

$$
\left|u_{1}\right| \leq \frac{\left|y\left(s_{1} ; t_{2}, x_{2}\right)-x_{2}\right|}{h_{1}}+\frac{\left|x_{2}-x_{1}\right|}{h_{1}} \leq 2 r+1 .
$$

Let $y_{1}:\left[0, t_{1}\right] \mapsto \mathbb{R}^{n}$ be the function defined by

$$
y_{1}(s)= \begin{cases}x_{1}+s u_{1} & \text { if } 0 \leq s \leq h_{1} \\ y\left(s-t_{1}+t_{2} ; t_{2}, x_{2}\right) & \text { if } h_{1} \leq s \leq t_{1}\end{cases}
$$


Then

$$
\begin{aligned}
V\left(t_{1}, x_{1}\right) & \leq \int_{0}^{t_{1}} L\left(y_{1}(s), y_{1}^{\prime}(s)\right) d s+\varphi\left(y_{1}\left(t_{1}\right)\right)=\int_{0}^{h_{1}} L\left(x_{1}+s u_{1}, u_{1}\right) d s \\
& +\int_{s_{1}}^{t_{2}} L\left(y\left(s ; t_{2}, x_{2}\right), y^{\prime}\left(s ; t_{2}, x_{2}\right)\right) d s+\varphi\left(y\left(t_{2} ; t_{2}, x_{2}\right)\right) .
\end{aligned}
$$

As $s_{1}=h_{1}-t_{1}+t_{2} \geq 0$ and $L \geq 0$, we obtain

$$
V\left(t_{1}, x_{1}\right) \leq \int_{0}^{h_{1}} L\left(x_{1}+s u_{1}, u_{1}\right) d s+V\left(t_{2}, x_{2}\right) .
$$

Since $L$ is locally bounded, it follows from (3.16) that there exists a constant $M$, depending only on $L, t_{0}, x_{0}$, $\delta$, and $r$, such that

$$
V\left(t_{1}, x_{1}\right)-V\left(t_{2}, x_{2}\right) \leq M h_{1}=M\left(\left|t_{1}-t_{2}\right|+\left|x_{1}-x_{2}\right|\right) .
$$

Exchanging the roles of $\left(t_{1}, x_{1}\right)$ and $\left(t_{2}, x_{2}\right)$ we obtain that $V$ in $M$-Lipschitz on $B\left(\left(t_{0}, x_{0}\right), \delta\right)$.

When $L$ is discontinuous, different contingent inequalities have to be introduced, which involve the function $L^{+}(x, u)$ defined by

$$
\begin{aligned}
L^{+}(x, u) & =\limsup _{h \rightarrow 0+} \frac{1}{h} \inf \left\{\int_{-h}^{0} L\left(y(s), y^{\prime}(s)\right) d s: y(-h)=x-h u, y(0)=x\right\} \\
& =\limsup _{h \rightarrow 0+} \frac{1}{h} \inf \left\{\int_{0}^{h} L\left(y(s),-y^{\prime}(s)\right) d s: y(0)=x, y(h)=x-h u\right\} .
\end{aligned}
$$

Remark 3.5. The function $L^{+}(x, u)$ was introduced in [1]. In that paper it was shown that, if for some positive constants $D, d$ and $p>1$ we have

$$
\forall(x, u) \in \mathbb{R}^{n} \times \mathbb{R}^{n}, \quad d|u|^{p} \leq L(x, u) \leq D\left(1+|u|^{p}\right),
$$

then $L^{+}(x, \cdot)$ is continuous for every $x \in \mathbb{R}^{n}$ and convex for almost every $x \in \mathbb{R}^{n}$.

Clearly for all $u$ the function $L^{+}(\cdot, u)$ is smaller than or equal to the upper semicontinuous envelope of $L(\cdot, u)$.

Proposition 3.6. If $L$ is locally bounded, then $L^{+}(x, u) \geq L(x, u)$ for all $(x, u) \in \mathbb{R}^{n} \times \mathbb{R}^{n}$. Moreover, if $v_{h} \rightarrow u$ as $h \rightarrow 0+$, then

$$
L^{+}(x, u)=\limsup _{h \rightarrow 0+} \frac{1}{h} \inf \left\{\int_{-h}^{0} L\left(y(s), y^{\prime}(s)\right) d s: y(-h)=x-h v_{h}, y(0)=x\right\} .
$$

In particular $L^{+}=L$ when $L$ is continuous.

Proof. Let us fix $(x, u)$ and $v_{h}$ as in the statement of the proposition, and let $\bar{L}(x, u)$ be the right hand side of the formula to be proved. We want to show that $\bar{L}(x, u) \leq L^{+}(x, u)$. For every $h>0$ let $\varepsilon_{h}=\left|v_{h}-u\right|$ and let $y_{h}$ be a minimizer of the problem

$$
\inf \left\{\int_{-\left(1-\varepsilon_{h}\right) h}^{0} L\left(y(s), y^{\prime}(s)\right) d s: y\left(-\left(1-\varepsilon_{h}\right) h\right)=x-\left(1-\varepsilon_{h}\right) h u, y(0)=x\right\} .
$$


For all $s \in\left[-h,-\left(1-\varepsilon_{h}\right) h\right]$ set

$$
y_{h}(s)=x-\left(1-\varepsilon_{h}\right) h u+\left(s+\left(1-\varepsilon_{h}\right) h\right) \frac{v_{h}-\left(1-\varepsilon_{h}\right) u}{\varepsilon_{h}} .
$$

Then $y_{h}(-h)=x-h v_{h}$. Since $\left(v_{h}-\left(1-\varepsilon_{h}\right) u\right) / \varepsilon_{h}$ is bounded, we deduce that for some $M>0$ and all $h>0$,

$$
\int_{-h}^{0} L\left(y_{h}(s), y_{h}^{\prime}(s)\right) d s \leq \int_{-\left(1-\varepsilon_{h}\right) h}^{0} L\left(y_{h}(s), y_{h}^{\prime}(s)\right) d s+\int_{-h}^{-\left(1-\varepsilon_{h}\right) h} M d s .
$$

Dividing by $h$ and taking the upper limit when $h \rightarrow 0+$ we get $\bar{L}(x, u) \leq L^{+}(x, u)$. The opposite inequality can be proved in the same way.

To prove that $L^{+}(x, u) \geq L(x, u)$, for every $h>0$ let $y_{h}$ be a minimizer of the problem

$$
\inf \left\{\int_{-h}^{0} L\left(y(s), y^{\prime}(s)\right) d s: y(-h)=x-h u, y(0)=x\right\} .
$$

Observe that $L^{+}(x, u)$ is finite, because $L$ is locally bounded. By [2] there exist $k_{h}$ such that for some $p_{h}(s) \in$ $\partial_{u} L\left(y_{h}(s), y_{h}^{\prime}(s)\right)$

$$
\left\langle p_{h}(s), y_{h}^{\prime}(s)\right\rangle-L\left(y_{h}(s), y_{h}^{\prime}(s)\right)=k_{h}
$$

We set $y_{h}(s)=y_{h}(-h)=x-h u$ for $s \leq-h$. Then for some $M \geq 0$

$$
\int_{-1}^{0} L\left(y_{h}(s), y_{h}^{\prime}(s)\right) d s \leq M
$$

Arguing as in the proof of Proposition 3.3, we deduce that the sequence $k_{h}$ is bounded. By [2] (proof of Th. 4.2) the sequence $y_{h}^{\prime}$ is bounded in $L^{\infty}$.

Let $\varepsilon>0$. Since $L$ is lower semicontinuous and continuous in the second variable, for all $h$ small enough

$$
L\left(y_{h}(s), y_{h}^{\prime}(s)\right) \geq L\left(x, y_{h}^{\prime}(s)\right)-\varepsilon \quad \text { a.e. in }[-h, 0]
$$

(see Step 2 in the proof of Prop. 3.2). Integrating and using Jensen's inequality we get

$$
\frac{1}{h} \int_{-h}^{0} L\left(y_{h}(s), y_{h}^{\prime}(s)\right) d s \geq \frac{1}{h} \int_{-h}^{0} L\left(x, y_{h}^{\prime}(s)\right) d s-\varepsilon \geq L(x, u)-\varepsilon .
$$

Taking the upper limit when $h \rightarrow 0+$ and $\varepsilon \rightarrow 0+$ we obtain $L^{+}(x, u) \geq L(x, u)$.

If $L$ is continuous, we have also $L^{+} \leq L$ by Remark 3.5, hence $L^{+}=L$.

We generalize now a result obtained in [1] under some additional growth conditions.

Proposition 3.7. Assume that $L$ is locally bounded. Let $y:[0, T] \mapsto \mathbb{R}^{n}$ be a Lipschitz function. Then $L\left(y(t), y^{\prime}(t)\right)=L^{+}\left(y(t), y^{\prime}(t)\right)$ for almost every $t \in[0, T]$.

Proof. We already know that $L^{+} \geq L$. To prove the opposite inequality, we fix a function $y$ as in the statement of the proposition. Since $L$ is locally bounded, the function $t \mapsto \psi(t):=\int_{0}^{t} L\left(y(s), y^{\prime}(s)\right) d s$ is absolutely continuous. Let $t \in[0, T]$ be such that both $\psi^{\prime}(t)$ and $y^{\prime}(t)$ do exist and $\psi^{\prime}(t)=L\left(y(t), y^{\prime}(t)\right)$. Since $v_{h}=(y(t)-y(t-h)) / h$ 
converges to $y^{\prime}(t)$ as $h \rightarrow 0+$, from Proposition 3.6 we obtain

$$
L^{+}\left(y(t), y^{\prime}(t)\right) \leq \lim _{h \rightarrow 0+} \frac{1}{h} \int_{t-h}^{t} L\left(y(s), y^{\prime}(s)\right) d s=\psi^{\prime}(t)=L\left(y(t), y^{\prime}(t)\right),
$$

which concludes the proof.

\section{The Hamilton-Jacobi equation}

In this section we suppose that $L$ is continuous and we consider three different notions of generalized solutions of the Hamilton-Jacobi equation (1.4). In Theorem 4.1 we prove that the value function $V$ defined by (3.1) is the unique lower semicontinuous solution of the Hamilton-Jacobi equation (1.4) in a suitable generalized sense, expressed in terms of contingent inequalities. Then we restrict our attention to locally Lipschitz solutions, and we look for uniqueness results for weaker (and more usual) notions of solutions. In Theorem 4.5 we prove that $V$ is the unique locally Lipschitz function which satisfies the Hamilton-Jacobi equation in the following generalized sense:

$$
\forall(t, x) \in \mathbb{R}_{+}^{\star} \times \mathbb{R}^{n}, \quad \forall\left(p_{t}, p_{x}\right) \in \partial_{-} V(t, x), p_{t}+H\left(x,-p_{x}\right)=0
$$

Finally, in Theorem 4.6, we prove that $V$ is the unique locally Lipschitz viscosity solution of (1.4).

Theorem 4.1. Assume that $L$ is continuous. Then $V$ is lower semicontinuous on $\mathbb{R}_{+} \times \mathbb{R}^{n}$ and solves the Hamilton-Jacobi equation (1.4) in the following sense:

$$
\begin{gathered}
\forall(t, x) \in \operatorname{dom}(V), t>0, \exists u \in \mathbb{R}^{n}, \quad D_{\uparrow} V(t, x)(-1, u) \leq-L(x, u), \\
\forall(t, x) \in \operatorname{dom}(V), \forall u \in \mathbb{R}^{n}, \quad D_{\downarrow} V(t, x)(1,-u) \leq L(x, u) .
\end{gathered}
$$

Let $W: \mathbb{R}_{+} \times \mathbb{R}^{n} \mapsto \mathbb{R}_{+} \cup\{+\infty\}$ be a lower semicontinuous function which satisfies the initial condition $W(0, \cdot)=$ $\varphi$. If $W$ is a supersolution of the Hamilton-Jacobi equation (1.4), in the sense that

$$
\forall(t, x) \in \operatorname{dom}(W), t>0, \exists u \in \mathbb{R}^{n}, \quad D_{\uparrow} W(t, x)(-1, u) \leq-L(x, u),
$$

then $W \geq V$ on $\mathbb{R}_{+} \times \mathbb{R}^{n}$. If $W$ is a subsolution of the Hamilton-Jacobi equation (1.4), in the sense that

$$
\forall(t, x) \in \operatorname{dom}(W), \forall u \in \mathbb{R}^{n}, \quad D_{\downarrow} W(t, x)(1,-u) \leq L(x, u),
$$

then $W \leq V$ on $\mathbb{R}_{+} \times \mathbb{R}^{n}$.

Therefore $V$ is the unique non negative lower semicontinuous function which satisfies the initial condition $V(0, \cdot)=\varphi$ and solves the Hamilton-Jacobi equation (1.4) in the sense of (4.1) and (4.2).

Proof. The lower semicontinuity of $V$ is proved in Proposition 3.1. Condition (4.1) follows from Proposition 3.2. Let us prove (4.2). Fix $(t, x) \in \operatorname{dom}(V)$ and $u \in \mathbb{R}^{n}$. Observe that for all $h>0$ and $v \in \mathbb{R}^{n}$ we have

$$
V(t+h, x-h v)-V(t, x) \leq \int_{0}^{h} L(x-s v, v) d s
$$

Dividing by $h$ and taking the upper limit when $h \rightarrow 0+$ and $v \rightarrow u$ we obtain (4.2). 
Let $W$ be as in the statement of the theorem. Assume that $W$ is a supersolution, i.e., $W$ satisfies (4.3). Let $(t, x) \in \operatorname{dom}(W), t>0$, and $\varepsilon>0$. By (2.2) and (4.3) there exist $h_{i} \rightarrow 0+$ and $u_{i} \rightarrow u$ such that

$$
W\left(t-h_{i}, x+h_{i} u_{i}\right) \leq W(t, x)-h_{i} L(x, u)+\varepsilon h_{i} .
$$

Since $L$ is continuous, for all sufficiently large $i$ we have

$$
W\left(t-h_{i}, x+h_{i} u_{i}\right)+\int_{0}^{h_{i}} L\left(x+s u_{i}, u_{i}\right) d s \leq W(t, x)+2 \varepsilon h_{i} .
$$

Consider the set $\mathcal{A}$ of all pairs $(\sigma, y) \in] 0, t] \times W^{1,1}\left(0, \sigma ; \mathbb{R}^{n}\right)$ such that

$$
y(0)=x \quad \& W(t-\sigma, y(\sigma))+\int_{0}^{\sigma} L\left(y(s), y^{\prime}(s)\right) d s \leq W(t, x)+2 \varepsilon \sigma .
$$

The set $\mathcal{A}$ is nonempty by the first part of the proof. We introduce the following partial order on $\mathcal{A}$ : we write $(\sigma, y) \ll(\tau, z)$ if $\sigma \leq \tau$ and $y$ is the restriction of $z$ to $[0, \sigma]$.

We claim that for every totally ordered subset $\mathcal{B}$ of $\mathcal{A}$ there exists $(\tau, z) \in \mathcal{A}$ such that $(\sigma, y) \ll(\tau, z)$ for every $(\sigma, y) \in \mathcal{B}$. Indeed let

$$
\tau=\sup _{(\sigma, y) \in \mathcal{B}} \sigma
$$

and consider a sequence $\left(\sigma_{i}, y_{i}\right) \in \mathcal{B}$ with $\sigma_{i} \rightarrow \tau$. Define $z:\left[0, \tau\left[\mapsto \mathbb{R}^{n}\right.\right.$ by setting $z(s)=y_{i}(s)$ whenever $s<\sigma_{i}$. As $\mathcal{B}$ is totally ordered, the function $z$ is well defined and, if $(\sigma, y) \in \mathcal{B}$ with $\sigma<\tau$, then $y$ coincides with the restriction of $z$ to $[0, \sigma]$.

Since $W \geq 0$ we deduce that for some $c$ and for all $i$

$$
\int_{0}^{t_{i}} L\left(y_{i}(s), y_{i}^{\prime}(s)\right) d s \leq c
$$

Set $y_{i}(s)=y_{i}\left(\sigma_{i}\right)$ for all $\left.\left.s \in\right] \sigma_{i}, \tau\right]$. Since $L$ satisfies the coercivity assumption (1.2), $y_{i}^{\prime}$ are equiintegrable on $[0, \tau]$. Consequently, $y_{i}$ are equicontinuous on $[0, \tau]$. So $z$ can be extended by continuity at $\tau$, $z$ belongs to $W^{1,1}\left(0, \tau ; \mathbb{R}^{n}\right)$ and $(\tau, z)$ belongs to $\mathcal{A}$ (recall that $W$ is lower semicontinuous). It is clear from the construction that $(\sigma, y) \ll(\tau, z)$ for every $(\sigma, y) \in \mathcal{B}$.

By Zorn's lemma there exists a maximal element $(\sigma, y) \in \mathcal{A}$. We claim that $\sigma=t$. Indeed, if not, then acting as at the beginning of the proof, we construct $(\tau, z) \in \mathcal{A}$ with $\sigma<\tau \leq t$ and $(\sigma, y) \ll(\tau, z)$, which contradicts the maximality. So

$$
V(t, x) \leq W(0, y(t))+\int_{0}^{t} L\left(y(s), y^{\prime}(s)\right) d s \leq W(t, x)+2 \varepsilon t .
$$

The number $\varepsilon>0$ being arbitrary, we conclude that $V(x, t) \leq W(x, t)$.

Suppose now that $W$ is a subsolution, i.e., $W$ satisfies (4.4). Let us fix $t>0$ and $x \in \mathbb{R}^{n}$, and let $y$ be a minimizer of (3.1). Since $L$ is continuous (1.3) holds true. Thus $y^{\prime} \in L^{\infty}\left(0, t ; \mathbb{R}^{n}\right)$ by [2]. Consider a sequence of continuous functions $u_{i}:[0, t] \mapsto \mathbb{R}^{n}$ which is bounded in $L^{\infty}\left(0, t ; \mathbb{R}^{n}\right)$ and converges to $y^{\prime}$ almost everywhere in $[0, t]$, and define

$$
y_{i}(s)=y(t)-\int_{s}^{t} u_{i}(\tau) d \tau
$$

Then $y_{i}$ converges to $y$ uniformly in $[0, t]$. Fix $i$ and set $\psi(s)=W\left(s, y_{i}(t-s)\right)$ for $0 \leq s \leq t$. By (4.4) for every $s \in \operatorname{dom}(\psi)$, with $s<t$, we have

$$
D_{\uparrow} \psi(s)(1) \leq L\left(y_{i}(t-s), u_{i}(t-s)\right)
$$


Consider the closed set

$$
K=\mathcal{E} p i(\psi) \cup([t,+\infty[\times \mathbb{R})
$$

and the system

$$
\left\{\begin{array}{l}
\left(\alpha^{\prime}(s), z^{\prime}(s)\right)=\left(1, L\left(y_{i}(t-s), u_{i}(t-s)\right)\right) \\
(\alpha(0), z(0))=(0, \varphi(y(t)))
\end{array}\right.
$$

It has the unique solution

$$
(\alpha(s), z(s))=\left(s, \varphi(y(t))+\int_{0}^{s} L\left(y_{i}(t-\tau), u_{i}(t-\tau)\right) d \tau\right) .
$$

According to (2.3), Theorem 2.3, and (4.5), this solution is viable in $K$, i.e., for all $s \in[0, t],(\alpha(s), z(s)) \in K$. Thus for all $s<t$

$$
W\left(s, y_{i}(t-s)\right) \leq \varphi(y(t))+\int_{0}^{t} L\left(y_{i}(\tau), u_{i}(\tau)\right) d \tau
$$

Since $L$ is continuous and $W$ is lower semicontinuous, from the Lebesgue Theorem we obtain

$$
W(t, x) \leq \varphi(y(t))+\int_{0}^{t} L\left(y(\tau), y^{\prime}(\tau)\right) d \tau
$$

which gives $W(t, x) \leq V(t, x)$.

Remark 4.2. In the previous theorem, the comparison result for subsolutions remains true, if we assume that (4.4) holds only for $t>0$, provided that $W$ satisfies also condition (4.20) of the next theorem. In this case (4.5) holds only for $s>0$, but we can modify the proof in the following way. We fix $t_{i} \rightarrow 0+$ and $x_{i} \rightarrow y(t)$ such that $W\left(t_{i}, x_{i}\right) \rightarrow \varphi(y(t))$, and define

$$
y_{i}(s)=x_{i}-\int_{s}^{t-t_{i}} u_{i}(\tau) d \tau
$$

for $0 \leq s \leq t-t_{i}$ Consequently, $\psi(s)$ is defined only for $t_{i} \leq s \leq t$, and (4.5) holds only for $t_{i} \leq s \leq t$. Then we replace 0 by $t_{i}$ and $\varphi(y(t))$ by $W\left(t_{i}, x_{i}\right)$ in (4.6-4.8), and we obtain (4.9) as before.

Remark 4.3. The following example shows that we can not remove the assumption $W \geq 0$ in Theorem 4.1 even if $L$ does not depend on $x$. Let $n=1, L(x, u)=\frac{1}{2}|u|^{2}, \varphi(x)=0$. Then $V(t, x)=0$ and $H(x, p)=\frac{1}{2}|p|^{2}$. Let us consider the lower semicontinuous function $W: \mathbb{R}_{+} \times \mathbb{R}^{n} \mapsto \mathbb{R}$ defined by

$$
W(t, x)= \begin{cases}0 & \text { if } t x<1 \\ -(t x-1)^{1 / 2}-x^{3}-x t^{-2} & \text { if } t x \geq 1 .\end{cases}
$$

By direct computation one checks that for $t x \neq 1$ the function $W$ is differentiable and

$$
W_{t}(t, x)+H\left(x,-W_{x}(t, x)\right)=W_{t}(t, x)+\frac{1}{2}\left|W_{x}(t, x)\right|^{2} \geq 0,
$$

which implies that

$$
\exists u \in \mathbb{R}^{n}, \quad D_{\uparrow} W(t, x)(-1, u) \leq-L(x, u) .
$$


On the other hand for $t x=1$ we have

$$
\forall u>1 / t^{2}, \quad D_{\uparrow} W(t, x)(-1, u)=-\infty,
$$

which implies (4.10) also in this case. Therefore $W$ satisfies $(4.3)$, but $W(t, x)<V(t, x)$ for $t x \geq 1$.

The following proposition shows the equivalence between the notion of subsolution considered in the previous theorem and a notion defined by using subdifferentials.

Proposition 4.4. Assume that $L$ is continuous. Let $W: \mathbb{R}_{+} \times \mathbb{R}^{n} \mapsto \mathbb{R} \cup\{+\infty\}$ be a lower semicontinuous function. Then the following conditions are equivalent:

$$
\begin{gathered}
\forall(t, x) \in \operatorname{dom}(W), \forall u \in \mathbb{R}^{n}, \quad D_{\downarrow} W(t, x)(1,-u) \leq L(x, u) ; \\
\forall(t, x) \in \operatorname{dom}(W), \forall u \in \mathbb{R}^{n}, \quad D_{\uparrow} W(t, x)(1,-u) \leq L(x, u) ; \\
\forall(t, x) \in \mathbb{R}_{+} \times \mathbb{R}^{n}, \quad \forall\left(p_{t}, p_{x}\right) \in \partial_{-} W(t, x), p_{t}+H\left(x,-p_{x}\right) \leq 0 .
\end{gathered}
$$

The equivalence remains true if $\operatorname{dom}(W)$ is replaced by $\operatorname{dom}(W) \cap\left(\mathbb{R}_{+}^{*} \times \mathbb{R}^{n}\right)$ in (4.11) and (4.12) and $\mathbb{R}_{+} \times \mathbb{R}^{n}$ is replaced by $\mathbb{R}_{+}^{\star} \times \mathbb{R}^{n}$ in (4.13).

Proof. It is clear that (4.11) implies (4.12). Suppose that $W$ satisfies (4.12). Then (4.13) follows from (2.4) and from the definition (1.5) of $H$.

Conversely, suppose that $W$ satisfies (4.13). We claim that for all $(t, x) \in \operatorname{dom}(W)$

$$
\forall u \in \mathbb{R}^{n}, \quad(1,-u, L(x, u)) \in \overline{c o} T_{\mathcal{E} p i(W)}(t, x, W(t, x)) .
$$

To prove this fact, let us fix $u \in \mathbb{R}^{n}$. Then

$$
\forall\left(p_{t}, p_{x}\right) \in \partial_{-} W(t, x), \quad p_{t}+\left\langle-p_{x}, u\right\rangle-L(x, u) \leq 0 .
$$

We want to prove that

$$
\forall\left(p_{t}, p_{x}, q\right) \in\left[T_{\mathcal{E} p i(W)}(t, x, W(t, x))\right]^{-}, p_{t}+\left\langle-p_{x}, u\right\rangle+q L(x, u) \leq 0 .
$$

When $q<0$ this inequality follows from (2.1) and (4.15). By Lemma 2.2, if $(0,0,0) \neq\left(p_{t}, p_{x}, 0\right) \in\left[T_{\mathcal{E} p i(W)}(t, x\right.$, $W(t, x))]^{-}$, then for some $\left(t_{i}, x_{i}\right) \rightarrow(t, x)$ and $\left(p_{t}^{i}, p_{x}^{i}, q_{i}\right) \in\left[T_{\mathcal{E} p i(W)}\left(t_{i}, x_{i}, W\left(t_{i}, x_{i}\right)\right)\right]^{-}$, with $q_{i}<0$, we have $\left(p_{t}^{i}, p_{x}^{i}, q_{i}\right) \rightarrow\left(p_{t}, p_{x}, 0\right)$. So

$$
p_{t}^{i}+\left\langle-p_{x}^{i}, u\right\rangle+q_{i} L\left(x_{i}, u\right) \leq 0 .
$$

Taking the limit we get $p_{t}+\left\langle-p_{x}, u\right\rangle \leq 0$, which concludes the proof of (4.16).

By the separation theorem (4.14) follows from (4.16). Thus for all $(t, x, r) \in \mathcal{E} p i(W)$

$$
(1,-u, L(x, u)) \in \overline{c o} T_{\mathcal{E} p i(W)}(t, x, r) .
$$

From Theorem 2.1 and continuity of $L$ we deduce that for all $(t, x) \in \operatorname{dom}(W)$,

$$
(1,-u, L(x, u)) \in C_{\mathcal{E} p i(W)}(t, x, W(t, x)) .
$$


Fix $\varepsilon>0$. Then it is not difficult to check that

$$
\forall u \in \mathbb{R}^{n}, \quad(1,-u, L(x, u)+\varepsilon) \in \operatorname{Int}\left(C_{\mathcal{E} p i(W)}(t, x, W(t, x))\right) .
$$

By [8] (Prop. 13, p. 425) this yields

$$
\limsup _{h \rightarrow 0+, v \rightarrow u} \frac{W(t+h, x-h v)-W(t, x)}{h} \leq L(x, u)+\varepsilon .
$$

As $\varepsilon>0$ is arbitrary, we obtain (4.11).

We recall that for a locally Lipschitz function $\varphi: \mathbb{R}^{n} \mapsto \mathbb{R}$ the contingent derivatives coincides with the Dini derivatives:

$$
\begin{aligned}
& \forall u \in \mathbb{R}^{n}, \quad D_{\uparrow} \varphi(x)(u)=d_{-} \varphi(x ; u):=\liminf _{h \rightarrow 0+} \frac{\varphi(x+h u)-\varphi(x)}{h}<+\infty . \\
& \forall u \in \mathbb{R}^{n}, \quad D_{\downarrow} \varphi(x)(u)=d_{+} \varphi(x ; u):=\limsup _{h \rightarrow 0+} \frac{\varphi(x+h u)-\varphi(x)}{h}<+\infty .
\end{aligned}
$$

By (2.3) this implies that

$$
(p, 0) \in\left[T_{\mathcal{E} p i(\varphi)}(x, \varphi(x))\right]^{-} \Longrightarrow p=0
$$

Theorem 4.5. Assume that $L$ is continuous. Then $V$ is locally Lipschitz on $\mathbb{R}_{+}^{\star} \times \mathbb{R}^{n}$ and solves the HamiltonJacobi equation (1.4) in the following sense:

$$
\forall(t, x) \in \mathbb{R}_{+}^{\star} \times \mathbb{R}^{n}, \quad \forall\left(p_{t}, p_{x}\right) \in \partial_{-} V(t, x), \quad p_{t}+H\left(x,-p_{x}\right)=0 .
$$

Let $W: \mathbb{R}_{+}^{\star} \times \mathbb{R}^{n} \mapsto \mathbb{R}_{+}$be a locally Lipschitz function which satisfies the initial condition

$$
\forall x \in \mathbb{R}^{n}, \quad \liminf _{h \rightarrow 0+, y \rightarrow x} W(h, y)=\varphi(x) .
$$

If $W$ is a supersolution of the Hamilton-Jacobi equation (1.4), in the sense that

$$
\forall(t, x) \in \mathbb{R}_{+}^{\star} \times \mathbb{R}^{n}, \quad \forall\left(p_{t}, p_{x}\right) \in \partial_{-} W(t, x), p_{t}+H\left(x,-p_{x}\right) \geq 0,
$$

then $W \geq V$ on $\mathbb{R}_{+}^{\star} \times \mathbb{R}^{n}$. If $W$ is a subsolution of the Hamilton-Jacobi equation (1.4), in the sense that

$$
\forall(t, x) \in \mathbb{R}_{+}^{\star} \times \mathbb{R}^{n}, \quad \forall\left(p_{t}, p_{x}\right) \in \partial_{-} W(t, x), p_{t}+H\left(x,-p_{x}\right) \leq 0,
$$

then $W \leq V$ on $\mathbb{R}_{+}^{\star} \times \mathbb{R}^{n}$.

Therefore the restriction of $V$ to $\mathbb{R}_{+}^{\star} \times \mathbb{R}^{n}$ is the unique non negative locally Lipschitz function which satisfies the initial condition (4.20) and solves the Hamilton-Jacobi equation (1.4) in the sense of (4.19).

Proof. The fact that $V$ is locally Lipschitz on $\mathbb{R}_{+}^{\star} \times \mathbb{R}^{n}$ is proved in Corollary 3.4. Property (4.19) follows from Theorem 4.1. Indeed (4.1) and (2.4), together with the definition (1.5) of $H$, imply that

$$
\forall(t, x) \in \mathbb{R}_{+} \times \mathbb{R}^{n}, \quad \forall\left(p_{t}, p_{x}\right) \in \partial_{-} V(t, x), \quad p_{t}+H\left(x,-p_{x}\right) \geq 0 ;
$$


the opposite inequality follows from (4.2) and Proposition 4.4. The initial condition (4.20) for $V$ is proved in Proposition 3.1 .

Let $W$ be as in the statement of the theorem. Assume that $W$ is a viscosity supersolution, i.e., $W$ satisfies (4.21). Define

$$
G(x)=\left\{(-1, u,-L(x, u)-\rho): \rho \geq 0, u \in \mathbb{R}^{n}\right\}
$$

and fix $t>0$ and $x \in \mathbb{R}^{n}$. Since $W$ is Lipschitz around $(t, x), \partial_{-} W$ is locally bounded. By the coercivity assumption (1.2) there exist $R>0$ and $\delta>0$ such that

$$
\forall(s, z) \in B((t, x), \delta), \quad \forall\left(p_{t}, p_{x}\right) \in \partial_{-} W(s, z), \quad \forall|u| \geq R, p_{t}+\left\langle-p_{x}, u\right\rangle-L(z, u)<0 .
$$

This (4.21), and the separation theorem imply that for every $(s, z) \in B((t, x), \delta)$

$$
G(z) \cap(\{-1\} \times B(0, R) \times[-m, 0]) \cap\left(\overline{c o} T_{\mathcal{E} p i(W)}(s, z, W(s, z))\right) \neq \emptyset
$$

where $m=\max \{L(z, u):(z, u) \in B(x, \delta) \times B(0, R)\}$. The above holds true also with $W(s, z)$ replaced by any $r \geq W(s, z)$. From Theorem 2.3 we obtain

$$
G(x) \cap(\{-1\} \times B(0, R) \times[-m, 0]) \cap\left(T_{\mathcal{E}_{p i(W)}}(t, x, W(t, x))\right) \neq \emptyset,
$$

which is equivalent to

$$
\exists u \in B(0, R), D_{\uparrow} W(t, x)(-1, u) \leq-L(x, u) .
$$

From Theorem 4.1 we deduce that $W \geq V$ on $\mathbb{R}_{+}^{\star} \times \mathbb{R}^{n}$.

If $W$ is a subsolution, i.e., $W$ satisfies $(4.22)$, then $W \leq V$ on $\mathbb{R}_{+}^{\star} \times \mathbb{R}^{n}$ by Theorem 4.1 and Remark 4.2.

Theorem 4.6. Assume that $L$ is continuous. Then $V$ is locally Lipschitz on $\mathbb{R}_{+}^{\star} \times \mathbb{R}^{n}$ and solves the HamiltonJacobi equation (1.4) in the viscosity sense, i.e.,

$$
\begin{aligned}
& \forall(t, x) \in \mathbb{R}_{+}^{\star} \times \mathbb{R}^{n}, \quad \forall\left(p_{t}, p_{x}\right) \in \partial_{-} V(t, x), p_{t}+H\left(x,-p_{x}\right) \geq 0, \\
& \forall(t, x) \in \mathbb{R}_{+}^{\star} \times \mathbb{R}^{n}, \quad \forall\left(p_{t}, p_{x}\right) \in \partial_{+} V(t, x), \quad p_{t}+H\left(x,-p_{x}\right) \leq 0 .
\end{aligned}
$$

Let $W: \mathbb{R}_{+}^{\star} \times \mathbb{R}^{n} \mapsto \mathbb{R}_{+}$be a locally Lipschitz function which satisfies the initial condition (4.20). If $W$ is a viscosity supersolution of the Hamilton-Jacobi equation (1.4), i.e.,

$$
\forall(t, x) \in \mathbb{R}_{+}^{\star} \times \mathbb{R}^{n}, \quad \forall\left(p_{t}, p_{x}\right) \in \partial_{-} W(t, x), \quad p_{t}+H\left(x,-p_{x}\right) \geq 0,
$$

then $W \geq V$ on $\mathbb{R}_{+}^{\star} \times \mathbb{R}^{n}$. If $W$ is a viscosity subsolution of the Hamilton-Jacobi equation (1.4), i.e.,

$$
\forall(t, x) \in \mathbb{R}_{+}^{*} \times \mathbb{R}^{n}, \quad \forall\left(p_{t}, p_{x}\right) \in \partial_{+} W(t, x), p_{t}+H\left(x,-p_{x}\right) \leq 0,
$$

then $W \leq V$ on $\mathbb{R}_{+}^{\star} \times \mathbb{R}^{n}$.

Therefore the restriction of $V$ to $\mathbb{R}_{+}^{\star} \times \mathbb{R}^{n}$ is the unique non negative locally Lipschitz viscosity solution of the Hamilton-Jacobi equation (1.4) which satisfies the initial condition (4.20). 
Proof. Let us prove (4.24). Fix $(t, x) \in \operatorname{dom}(V), t>0$, and $u \in \mathbb{R}^{n}$. Observe that for all small $h>0$ we have

$$
V(t, x) \leq V(t-h, x+h u)+\int_{0}^{h} L(x+s u, u) d s,
$$

hence

$$
V(t-h, x+h u))-V(t, x) \geq-\int_{0}^{h} L(x+s u, u) d s .
$$

Dividing by $h$ and taking the upper limit when $h \rightarrow 0+$ we obtain

$$
D_{\downarrow} V(t, x)(-1, u) \geq-L(x, u),
$$

which implies (4.24) by (2.5) and by the definition (1.5) of $H$. For the other properties of $V$ see Theorem 4.5.

Let $W$ be as in the statement of the theorem. If $W$ is a viscosity supersolution, i.e., $W$ satisfies (4.25), then $W \geq V$ on $\mathbb{R}_{+}^{\star} \times \mathbb{R}^{n}$ by Theorem 4.5.

Assume now that $W$ is a viscosity subsolution, i.e., $W$ satisfies (4.26). Properties (2.1) and (4.18) imply that, for all $t>0$ and $x \in \mathbb{R}^{n}$,

$$
\left[T_{\mathcal{E} p i(-W)}(t, x,-W(t, x))\right]^{-}=\bigcup_{\lambda \geq 0} \lambda\left(-\partial_{+} W(t, x),-1\right) .
$$

Using the separation theorem, from (4.26) we obtain

$$
\forall u \in \mathbb{R}^{n}, \quad(-1, u, L(x, u)) \in \overline{\mathrm{co}} T_{\mathcal{E} p i(-W)}(t, x,-W(t, x)) .
$$

The above holds true also with $-W(t, x)$ replaced by any $r \geq-W(t, x)$. So, by Theorem 2.1 , for all $t>0$ and $x \in \mathbb{R}^{n}$,

$$
\forall u \in \mathbb{R}^{n}, \quad(-1, u, L(x, u)) \in T_{\mathcal{E} p i(-W)}(t, x,-W(t, x)),
$$

and therefore by $(2.3)$ and $(2.4)$

$$
\forall u \in \mathbb{R}^{n}, \quad D_{\downarrow} W(t, x)(-1, u) \geq-L(x, u) .
$$

Fix $t>0, x \in \mathbb{R}^{n}$ and let $y$ be a solution to (3.1). By [2] it is Lipschitz. Consider $\left(t_{i}, x_{i}\right) \rightarrow(0+, y(t))$ such that $\lim _{i \rightarrow \infty} W\left(t_{i}, x_{i}\right)=\varphi(y(t))$. Set

$$
y_{i}(s)=y(s)+x_{i}-y\left(t-t_{i}\right)
$$

The function $\psi(s):=W\left(t-s, y_{i}(s)\right)$ is locally Lipschitz on $\left[0, t\left[\right.\right.$. Fix $0 \leq s<t$ such that $\psi^{\prime}(s)$ and $y^{\prime}(s)$ do exist. Using the fact that $W$ is locally Lipschitz we get

$$
\psi^{\prime}(s)=D_{\downarrow} W\left(t-s, y_{i}(s)\right)\left(-1, y^{\prime}(s)\right) \geq-L\left(y_{i}(s), y_{i}^{\prime}(s)\right) .
$$

Consequently for every $0 \leq s<t$

$$
W\left(t-s, y_{i}(s)\right)-W\left(t, y_{i}(0)\right)=\psi(s)-\psi(0) \geq-\int_{0}^{s} L\left(y_{i}(\tau), y_{i}^{\prime}(\tau)\right) d \tau,
$$


and thus

$$
W\left(t_{i}, x_{i}\right)+\int_{0}^{t} L\left(y_{i}(\tau), y^{\prime}(\tau)\right) d \tau \geq W\left(t, y_{i}(0)\right)
$$

Passing to the limit when $i \rightarrow \infty$ and using continuity of $L$ we deduce that $W(t, x) \leq V(t, x)$.

\section{The CASe of a Discontinuous Lagrangian}

In this section we do not assume that $L$ is continuous. We can still prove (Th. 5.1) that the value function $V$ defined by (3.1) is the unique non negative lower semicontinuous solution of the Hamilton-Jacobi equation (1.4), but now we have to consider a weaker notion of generalized solution, which involves a contingent inequality for the function $L^{+}$introduced in (3.17). To prove uniqueness in the smaller class of locally Lipschitz functions we can use an even weaker notion of solution, where the contingent inequality (5.1) for supersolutions is replaced by a viscosity inequality (Th. 5.5).

Theorem 5.1. Assume that $L$ is locally bounded. Then $V$ is lower semicontinuous on $\mathbb{R}_{+} \times \mathbb{R}^{n}$ and solves the Hamilton-Jacobi equation (1.4) in the following sense:

$$
\begin{gathered}
\forall(t, x) \in \operatorname{dom}(V), t>0, \exists u \in \mathbb{R}^{n}, \quad D_{\uparrow} V(t, x)(-1, u) \leq-L(x, u), \\
\forall(t, x) \in \operatorname{dom}(V), \forall u \in \mathbb{R}^{n}, \quad D_{\downarrow} V(t, x)(1,-u) \leq L^{+}(x, u) .
\end{gathered}
$$

Let $W: \mathbb{R}_{+} \times \mathbb{R}^{n} \mapsto \mathbb{R}_{+} \cup\{+\infty\}$ be a lower semicontinuous function which satisfies the initial condition $W(0, \cdot)=$ $\varphi$. If $W$ is a supersolution of the Hamilton-Jacobi equation (1.4), in the sense that

$$
\forall(t, x) \in \operatorname{dom}(W), t>0, \exists u \in \mathbb{R}^{n}, \quad D_{\uparrow} W(t, x)(-1, u) \leq-L(x, u),
$$

then $W \geq V$ on $\mathbb{R}_{+} \times \mathbb{R}^{n}$. If $W$ is a subsolution of the Hamilton-Jacobi equation (1.4), in the sense that

$$
\forall(t, x) \in \operatorname{dom}(W), \forall u \in \mathbb{R}^{n}, \quad D_{\downarrow} W(t, x)(1,-u) \leq L^{+}(x, u),
$$

then $W \leq V$ on $\mathbb{R}_{+} \times \mathbb{R}^{n}$.

Therefore $V$ is the unique non negative lower semicontinuous function which satisfies the initial condition $V(0, \cdot)=\varphi$ and solves the Hamilton-Jacobi equation (1.4) in the sense of (5.1) and (5.2).

To prove the theorem, we need the following approximation lemma:

Lemma 5.2. There exists a sequence of continuous functions $L_{k}: \mathbb{R}^{n} \times \mathbb{R}^{n} \mapsto \mathbb{R}_{+}$, converging pointwise to L, such that, for all $k, L_{k}(x, \cdot)$ is convex, $L_{k} \leq L_{k+1} \leq L$, and

$$
\forall x \in \mathbb{R}^{n}, \forall u \in \mathbb{R}^{n}, \quad L_{k}(x, u) \geq \Theta(u) .
$$

For every $k$ let $V_{k}$ be the value function of problem (3.1) with $L$ replaced by $L_{k}$. Then $V_{k}$ converge to $V$ pointwise.

Proof. The proof of the existence of a sequence $L_{k}$ with the required properties can be found in [12] (Lem. 2.2.3). It is clear that the sequence $V_{k}$ is nondecreasing, so it is pointwise convergent, and that $V_{k} \leq V$. We want to prove that $V \leq \lim _{k} V_{k}$. Let us fix $(t, x)$ in $\mathbb{R}_{+} \times \mathbb{R}^{n}$ such that

$$
\lim _{k \rightarrow \infty} V_{k}(x, t)<+\infty
$$


Let $y_{k}$ be a solution to the problem

$$
\inf \left\{\int_{0}^{t} L_{k}\left(y(s), y^{\prime}(s)\right) d s+\varphi(y(t)): y(0)=x\right\} .
$$

We deduce from (5.6) and from the coercivity assumption (1.2) that $y_{k}^{\prime}$ are equiintegrable. Hence $y_{k}$ are equicontinuous. Taking a subsequence and keeping the same notations we may assume that $y_{k}$ converges uniformly to some $y$ and $y_{k}^{\prime}$ converges weakly in $L^{1}\left(0, t ; \mathbb{R}^{n}\right)$ to $y^{\prime}$. Fix $i$. Since for every $k \geq i$ we have

$$
\varphi\left(y_{k}(t)\right)+\int_{0}^{t} L_{i}\left(y_{k}(s), y_{k}^{\prime}(s)\right) d s \leq \varphi\left(y_{k}(t)\right)+\int_{0}^{t} L_{k}\left(y_{k}(s), y_{k}^{\prime}(s)\right) d s=V_{k}(t, x),
$$

taking the limit when $k \rightarrow \infty$ we get

$$
\varphi(y(t))+\int_{0}^{t} L_{i}\left(y(s), y^{\prime}(s)\right) d s \leq \lim _{k \rightarrow \infty} V_{k}(t, x) \leq V(t, x) .
$$

Taking the limit when $i \rightarrow \infty$ and using Fatou's lemma we deduce that

$$
V(t, x) \leq \varphi(y(t))+\int_{0}^{t} L\left(y(s), y^{\prime}(s)\right) d s \leq \lim _{k \rightarrow \infty} V_{k}(t, x) \leq V(t, x) .
$$

Thus $V_{k}$ converges pointwise to $V$.

Proof of Theorem 5.1. The lower semicontinuity of $V$ is proved in Proposition 3.1. Condition (5.1) follows from Proposition 3.2. Let us prove (5.2). Fix $(t, x) \in \operatorname{dom}(V)$ and $u \in \mathbb{R}^{n}$. By Proposition 3.6 we have

$$
L^{+}(x, u)=\limsup _{\substack{h \rightarrow 0+\\ v \rightarrow u}} \frac{1}{h} \inf \left\{\int_{-h}^{0} L\left(y(s), y^{\prime}(s)\right) d s: y(-h)=x-h v, y(0)=x\right\} .
$$

Since for every absolutely continuous function $y$ satisfying $y(-h)=x-h v$ and $y(0)=x$ we have

$$
V(t+h, x-h v) \leq V(t, x)+\int_{-h}^{0} L\left(y(s), y^{\prime}(s)\right) d s,
$$

we deduce that

$$
V(t+h, x-h v)-V(t, x) \leq \inf \left\{\int_{-h}^{0} L\left(y(s), y^{\prime}(s)\right) d s: y(0)=x, y(-h)=x-h v\right\} .
$$

Dividing by $h$ and taking the upper limit as $h \rightarrow 0+$ and $v \rightarrow u$, we obtain (5.2).

Let $W$ be as in the statement of the theorem. Assume that $W$ is a supersolution, in the sense that (5.3) is satisfied. Let $L_{k}$ and $V_{k}$ be the continuous Lagrangians and the corresponding value functions given by Lemma 5.2. As $L_{k} \leq L$, the function $W$ is a supersolution for the problem relative to the continuous Lagrangian $L_{k}$. Therefore $W \geq V_{k}$ on $\mathbb{R}_{+} \times \mathbb{R}^{n}$ by Theorem 4.1. Since $V_{k}$ converges to $V$ pointwise, we conclude that $W \geq V$ on $\mathbb{R}_{+} \times \mathbb{R}^{n}$.

Assume now that $W$ is a subsolution, i.e., $W$ satisfies (5.4). Fix $t>0, x \in \mathbb{R}^{n}$ and let $y$ be a minimizer of (3.1). It is Lipschitz continuous by [2]. Set $\psi(s)=W(s, y(t-s))$. Thus for almost all $s \in[0, t]$,

$$
D_{\uparrow} \psi(s)(1) \leq D_{\downarrow} W(s, y(t-s))\left(1,-y^{\prime}(t-s)\right) \leq L^{+}\left(y(t-s), y^{\prime}(t-s)\right) .
$$


By Proposition 3.7 we have

$$
L^{+}\left(y(t-s), y^{\prime}(t-s)\right)=L\left(y(t-s), y^{\prime}(t-s)\right) \text { a.e. in }[0, t]
$$

Since $y$ is Lipschitz and $L$ is locally bounded, the same argument used for (5.7) implies that there exists a constant $M$ such that

$$
\forall s \in\left[0, t\left[, \quad D_{\uparrow} \psi(s)(1) \leq M\right.\right.
$$

Define the closed set-valued map $s \rightsquigarrow P(s)$ by

$$
P(s)=W(s, y(t-s))+\mathbb{R}_{+}, \forall s \in[0, t[\quad \& \quad P(s)=\mathbb{R}, \forall s \geq t .
$$

Using $(5.9,2.3)$, and Theorem 2.3, we deduce that for every $s_{0} \in[0, t]$ and for every $z_{0} \in P\left(s_{0}\right)$ there exists an $M$-Lipschitz function $z:\left[s_{0}, t\right] \mapsto \mathbb{R}^{n}$ such that $z\left(s_{0}\right)=z_{0}$ and $z(s) \in P(s)$ for every $s \in\left[s_{0}, t\right]$. This yields that $P$ is left absolutely continuous on $[0, t]$, i.e., for any $\varepsilon>0$ and for any compact subset $K \subset \mathbb{R}^{n}$, there exists $\delta>0$ such that for any subdivision $0 \leq t_{1}<\tau_{1} \leq \ldots t_{m}<\tau_{m} \leq t$ with $\sum_{i}\left(\tau_{i}-t_{i}\right) \leq \delta$ we have $\sum_{i} h\left(P\left(t_{i}\right) \cap K, P\left(\tau_{i}\right)\right) \leq \varepsilon$, where $h$ is the Hausdorff semidistance: $h(A, B):=\sup _{a \in A} d(a, B)$.

Consider the viability problem

$$
\left\{\begin{array}{l}
z^{\prime}(s)=L\left(y(t-s), y^{\prime}(t-s)\right) \\
z(0)=\varphi(y(t)) \\
z(s) \in P(s)
\end{array}\right.
$$

According to the measurable viability theorem ([22], Th. 4.2) it has a (viable) solution by (5.7) and (5.8). But this solution is given by

$$
z(s)=\varphi(y(t))+\int_{0}^{s} L\left(y(t-\tau), y^{\prime}(t-\tau)\right) d \tau .
$$

Thus

and $W \leq V$ on $\mathbb{R}_{+} \times \mathbb{R}^{n}$.

$$
W(t, x) \leq \varphi(y(t))+\int_{0}^{t} L\left(y(\tau), y^{\prime}(\tau)\right) d \tau=V(t, x)
$$

The proof shows that the comparison result for supersolutions in Theorem 5.1 remains true even if we drop the assumption that $L$ is locally bounded. Therefore Proposition 3.2 and Theorem 5.1 imply the following corollary.

Corollary 5.3. Assume that $L$ satisfies (1.3). Then $V$ is the smallest non negative lower semicontinuous function satisfying the initial condition $V(0, \cdot)=\varphi$ and the contingent inequality (5.3).

Remark 5.4. In Theorem 5.1 the comparison result for subsolutions remains true, if we assume only that (5.4) holds for $t>0$, provided that $W$ satisfies condition (5.13) of the next theorem. In this case (5.9) holds only for $s>0$, but, to obtain the result, it is enough to replace 0 by $h>0$ and $\varphi(y(t))$ by $W(h, y(t-h))$ in $(5.10)$.

We consider now the uniqueness in the class of locally Lipschitz solutions.

Theorem 5.5. Assume that $L$ is locally bounded. Then $V$ is locally Lipschitz on $\mathbb{R}_{+}^{\star} \times \mathbb{R}^{n}$ and solves the Hamilton-Jacobi equation (1.4) in the following sense:

$$
\forall(t, x) \in \mathbb{R}_{+}^{\star} \times \mathbb{R}^{n}, \quad \forall\left(p_{t}, p_{x}\right) \in \partial_{-} V(t, x), \quad p_{t}+H\left(x,-p_{x}\right) \geq 0,
$$




$$
\forall(t, x) \in \mathbb{R}_{+}^{\star} \times \mathbb{R}^{n}, \quad \forall u \in \mathbb{R}^{n}, \quad D_{\downarrow} V(t, x)(1,-u) \leq L^{+}(x, u) .
$$

Let $W: \mathbb{R}_{+}^{\star} \times \mathbb{R}^{n} \mapsto \mathbb{R}_{+}$be a locally Lipschitz function which satisfies the initial condition (4.20) together with

$$
\forall x \in \mathbb{R}^{n}, \forall \lambda>0, \lim _{\substack{h \rightarrow 0+, y \rightarrow x \\|y-x| \leq \lambda h}} W(h, y)=\varphi(x) .
$$

If $W$ is a supersolution of the Hamilton-Jacobi equation (1.4), in the sense that

$$
\forall(t, x) \in \mathbb{R}_{+}^{*} \times \mathbb{R}^{n}, \quad \forall\left(p_{t}, p_{x}\right) \in \partial_{-} W(t, x), p_{t}+H\left(x,-p_{x}\right) \geq 0,
$$

then $W \geq V$ on $\mathbb{R}_{+}^{\star} \times \mathbb{R}^{n}$. If $W$ is a subsolution of the Hamilton-Jacobi equation (1.4), in the sense that

$$
\forall(t, x) \in \mathbb{R}_{+}^{*} \times \mathbb{R}^{n}, \quad \forall u \in \mathbb{R}^{n}, \quad D_{\downarrow} W(t, x)(1,-u) \leq L^{+}(x, u),
$$

then $W \leq V$ on $\mathbb{R}_{+}^{\star} \times \mathbb{R}^{n}$.

Therefore the restriction of $V$ to $\mathbb{R}_{+}^{\star} \times \mathbb{R}^{n}$ is the unique non negative locally Lipschitz function which satisfies the initial conditions (4.20) and (5.13) and solves the Hamilton-Jacobi equation (1.4) in the sense of (5.11) and (5.12).

Proof. The fact that $V$ is locally Lipschitz on $\mathbb{R}_{+}^{\star} \times \mathbb{R}^{n}$ is proved in Corollary 3.4. Conditions (4.20) and (5.13) for $V$ follow from Proposition 3.1. Condition (5.11) is proved in Proposition 3.2, while (5.12) follows from Theorem 5.1.

Let $W$ be as in the statement of the theorem. If $W$ is a supersolution, i.e., $W$ satisfies (5.14), then we can prove that $W \geq V$, arguing as in the proof of Theorem 4.5 (with Th. 4.1 replaced by Th. 5.1 ). If $W$ is a subsolution, i.e., $W$ satisfies (5.15), then $W \leq V$ by Theorem 5.1 and Remark 5.4.

\section{REFERENCES}

[1] M. Amar, G. Bellettini and S. Venturini, Integral representation of functionals defined on curves of $W^{1, p}$. Proc. Roy. Soc. Edinburgh Sect. A 128 (1998) 193-217.

[2] L. Ambrosio, O. Ascenzi and G. Buttazzo, Lipschitz regularity for minimizers of integral functionals with highly discontinuous integrands. J. Math. Anal. Appl. 142 (1989) 301-316.

[3] J.-P. Aubin, Contingent derivatives of set-valued maps and existence of solutions to nonlinear inclusions and differential inclusions. Advances in Mathematics, Supplementary Studies, edited by L. Nachbin (1981) 160-232.

[4] J.-P. Aubin, A survey of viability theory. SIAM J. Control Optim. 28 (1990) 749-788.

[5] J.-P. Aubin, Viability Theory. Birkhäuser, Boston (1991).

[6] J.-P. Aubin, Optima and Equilibria. Springer-Verlag, Berlin, Grad. Texts in Math. 140 (1993).

[7] J.-P. Aubin and A. Cellina, Differential Inclusions. Springer-Verlag, Berlin, Grundlehren Math. Wiss. 264 (1984).

[8] J.-P. Aubin and I. Ekeland, Applied Nonlinear Analysis. Wiley \& Sons, New York (1984).

[9] J.-P. Aubin and H. Frankowska, Set-Valued Analysis. Birkhäuser, Boston (1990).

[10] E.N. Barron and R. Jensen, Semicontinuous viscosity solutions for Hamilton-Jacobi equations with convex Hamiltonian. Comm. Partial Differential Equations 15 (1990) 1713-1742.

[11] J.W. Bebernes and J.D. Schuur, The Ważewski topological method for contingent equations. Ann. Mat. Pura Appl. 87 (1970) 271-280.

[12] G. Buttazzo, Semicontinuity, Relaxation and Integral Representation Problems in the Calculus of Variations. Longman, Harlow, Pitman Res. Notes Math. Ser. (1989).

[13] L. Cesari, Optimization Theory and Applications. Problems with Ordinary Differential Equations. Springer-Verlag, Berlin, Appl. Math. 17 (1983).

[14] B. Cornet, Regular properties of tangent and normal cones. Cahiers de Maths. de la Décision No. 8130 (1981).

[15] M.G. Crandall, P.-L. Lions, Viscosity solutions of Hamilton-Jacobi equations. Trans. Amer. Math. Soc. 277 (1983) 1-42.

[16] G. Dal Maso and L. Modica, Integral functionals determined by their minima. Rend. Sem. Mat. Univ. Padova 76 (1986) 255-267.

[17] C. Dellacherie, P.-A. Meyer, Probabilités et potentiel. Hermann, Paris (1975). 
[18] H. Frankowska, L'équation d'Hamilton-Jacobi contingente. C. R. Acad. Sci. Paris Sér. I Math. 304 (1987) $295-298$.

[19] H. Frankowska, Optimal trajectories associated to a solution of contingent Hamilton-Jacobi equations. Appl. Math. Optim. 19 (1989) 291-311.

[20] H. Frankowska, Lower semicontinuous solutions of Hamilton-Jacobi-Bellman equations, in Proc. of IEEE CDC Conference. Brighton, England (1991).

[21] H. Frankowska, Lower semicontinuous solutions of Hamilton-Jacobi-Bellman equations. SIAM J. Control Optim. 31 (1993) 257-272.

[22] H. Frankowska, S. Plaskacz and T. Rzeżuchowski, Measurable viability theorems and Hamilton-Jacobi-Bellman equation. J. Differential Equations 116 (1995) 265-305.

[23] G.N. Galbraith, Extended Hamilton-Jacobi characterization of value functions in optimal control. Preprint Washington University, Seattle (1998).

[24] H.G. Guseinov, A.I. Subbotin and. V.N. Ushakov, Derivatives for multivalued mappings with application to game-theoretical problems of control. Problems Control Inform. 14 (1985) 155-168.

[25] A.D. Ioffe, On lower semicontinuity of integral functionals. SIAM J. Control Optim. 15 (1977) 521-521 and 991-1000.

[26] C. Olech, Weak lower semicontinuity of integral functionals. J. Optim. Theory Appl. 19 (1976) 3-16.

[27] T. Rockafellar, Proximal subgradients, marginal values and augmented Lagrangians in nonconvex optimization. Math. Oper. Res. 6 (1981) 424-436.

[28] T. Rockafellar and R. Wets, Variational Analysis. Springer-Verlag, Berlin, Grundlehren Math. Wiss. 317 (1998).

[29] A.I. Subbotin, A generalization of the basic equation of the theory of the differential games. Soviet. Math. Dokl. 22 (1980) 358-362. 